\title{
BUNCHED BEAM STOCHASTIC COOLING IN THE FERMILAB RECYCLER RING*
}

\author{
D. Broemmelsiek, A. Burov, S. Nagaitsev, D. Neuffer ${ }^{\#}$, Fermilab, Batavia, IL 60439, U.S.A.
}

\section{Abstract}

Stochastic cooling with bunched beam in a linear bucket has been obtained and implemented operationally in the Fermilab Recycler Ring (RR). This is the first time that linear-rf bunched-beam stochastic cooling has been successfully used operationally in a high-energy facility. In this implementation the particle bunch length is much greater than the cooling system wavelengths, and that property is critical to the cooling success. The simultaneous longitudinal bunching enables cooling to much smaller longitudinal emittances than the coasting beam or barrier bucket system. Characteristics and limitations of bunched beam stochastic cooling are discussed.

\section{INTRODUCTION}

Prior to the RR, the Fermilab experience was that bunched beam cooling did not work, or at least not very well. A transverse cooling system was constructed for the Tevatron Collider, and was designed to cool Tevatron bunches during collision conditions. The system provided very little or no cooling, with the pickup signals dominated by coherent oscillations. Similar attempts in the SppS collider were also unsuccessful. (Cooling with full low-frequency rf buckets had been demonstrated in CERN ICE and the Fermilab AA rings.)

The ICE and AA cases had a large synchrotron frequency spread, and the synchrotron frequency spread enables the particle mixing needed for stochastic cooling. Bunched-beam cooling cases with small synchrotron frequency spread were expected to be unsuccessful.

In this paper we present results from recent experience in the RR that demonstrate useful bunched beam cooling. The successful Recycler cooling has a linear rf bucket that has no synchrotron frequency spread (no $\delta p / p$ dependence). In this paper we present experimental observations of the cooling. This is followed by discussions of the cooling data, of operational bunchedbeam cooling, and of future studies.

\section{EXPERIMENTAL STUDY}

In this section we present results of an experimental study where cooling in a barrier bucket and in a linear-rf bucket were directly compared. Parameters of the Recycler Ring are displayed in Table 1. For the experiment, the intensity was $\sim 30 \times 10^{10}$ antiprotons.

The beam was first cooled in a barrier bucket that was $\sim 4.8 \mu$ s long and $2 \mathrm{kV}$ in height. The beam was stochastically cooled in energy spread $\sigma_{\mathrm{E}}$ from $\sim 4 \mathrm{MeV}$ to

*Work supported by the US Department of energy under contract No. DE-AC072-76CH0300.

\#neuffer@fnal.gov $\sim 3 \mathrm{MeV}$. Experimental data is shown in fig. 1. The transverse emittance in these examples was measured to be $3 \pi \mathrm{mm}-\mathrm{mrad}$ (95\% emittances) after some initial cooling.

The cooling process can be parameterized in terms of the change of energy spread:

$$
\sigma_{\mathrm{E}}^{2}=\left(\sigma_{0}^{2}-\frac{\mathrm{D}}{2 \lambda}\right) \mathrm{e}^{-2 \lambda t}+\frac{\mathrm{D}}{2 \lambda}
$$

For the barrier-bucket beam data the fitted values are: $\sigma_{0}=$ 4.05 MeV, $\lambda=1.089$ hour $^{-1}, \mathrm{D}=17.53 \mathrm{MeV}^{2} /$ hour. Final equilibrium energy spread is $\sigma_{\mathrm{E} \text {,final }}=(\mathrm{D} / 2 \lambda)^{1 / 2}=2.84 \mathrm{MeV}$. This change in momentum spread reduces emittance by a corresponding factor; the longitudinal emittance cools from $\sim 82$ to $55 \mathrm{eV}$-s. If we use the approximately correct formula for emittance:

$$
\varepsilon_{\mathrm{BB}}=4 \sigma_{\mathrm{E}} \Delta \tau_{\text {full }},
$$

the emittance can then be written as:

$\varepsilon_{\mathrm{BB}}=\sqrt{\left(\varepsilon_{0}^{2}-\frac{8 \mathrm{D}\left(\Delta \tau_{\text {full }}\right)^{2}}{\lambda}\right) \mathrm{e}^{-2 \lambda \mathrm{t}}+\frac{8 \mathrm{D}\left(\Delta \tau_{\text {full }}\right)^{2}}{\lambda}}$.

A comparison of measured and calculated emittances for barrier bucket cooling is shown in fig. 1 .

Figure 1. Results of barrier bucket cooling measurement.

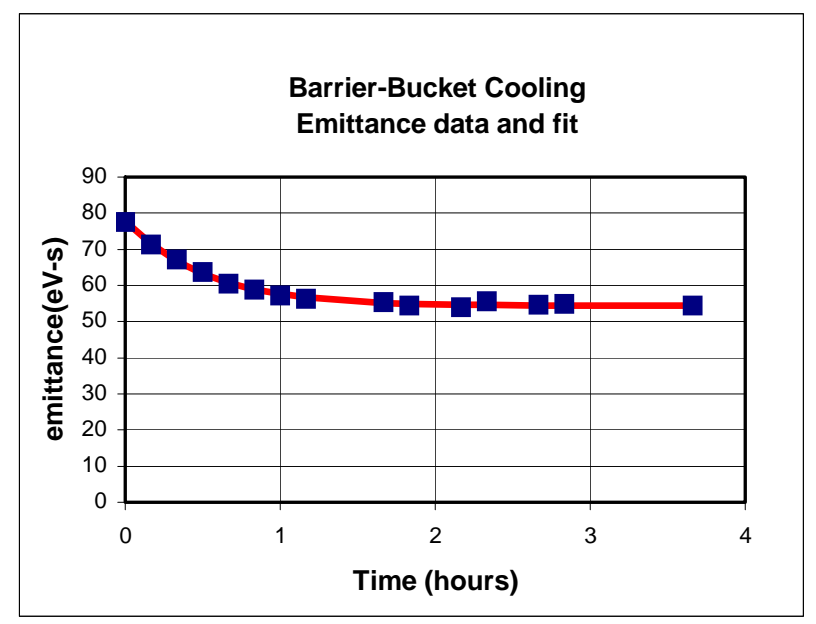

After the beam had reached an equilibrium emittance, the beam was adiabatically placed within a linear bucket. The rf gradient was $\sim 1 \mathrm{kV} / 2.4 \mu \mathrm{s}=0.417 \mathrm{kV} / \mu \mathrm{s}$. At these parameters the factor $\beta_{\tau \mathrm{E}}$ is $\sim 0.16 \mu \mathrm{s} / \mathrm{MeV}\left(\sigma_{\tau}=\beta_{\tau \mathrm{E}} \sigma_{\mathrm{E}}\right)$, and the synchrotron period $T_{\mathrm{s}}$ is $\sim 1.05 \mathrm{~s}$. In this bunching the energy spread increased to $\sim 4.6 \mathrm{MeV}$. The beam was again cooled until a final energy spread was obtained. (The transverse emittance increased by a small amount before reaching equilibrium. )

The linear-bunch cooling process can also be empirically parameterized in terms of the change of energy spread, as in equation 1 , but with the exponential 
growth a factor of 2 smaller because of the mixing between energy and phase coordinates. The emittance can be written as:

$$
\varepsilon_{L, \mathrm{rf}}(\mathrm{t})=6 \pi \beta_{\tau \mathrm{E}}\left[\left(\sigma_{0}^{2}-\frac{\mathrm{D}}{2 \lambda}\right) \mathrm{e}^{-\lambda \mathrm{t}}+\frac{\mathrm{D}}{2 \lambda}\right]
$$

with $\sigma_{0}=4.62 \mathrm{MeV}, \lambda=0.90$ hour $^{-1}, \mathrm{D}=14.05 . \lambda$ and $\mathrm{D}$ were $\sim 0.8 \times$ as large as that for the barrier bucket; the final equilibrium energy spread $\sigma_{\mathrm{E}, \text { final }}=(\mathrm{D} / 2 \lambda)^{1 / 2}=2.80$ $\mathrm{MeV}$, was barrier bucket. With linear bunching, the reduction in energy spread implies a concurrent reduction in bunch length (since $\sigma_{\tau}=\beta_{\tau \mathrm{E}} \sigma_{\mathrm{E}}$ ), from $\sigma_{\tau}=0.72$ to 0.45 $\mu$ s. The $6 \pi \sigma_{\mathrm{E}} \sigma_{\tau}$ emittance decreases from $\sim 65$ to $25 \mathrm{eV}$-s. Experimental results are displayed in fig. 2.

Figure 2: Linear bucket cooling: measured emitttances and model fit.

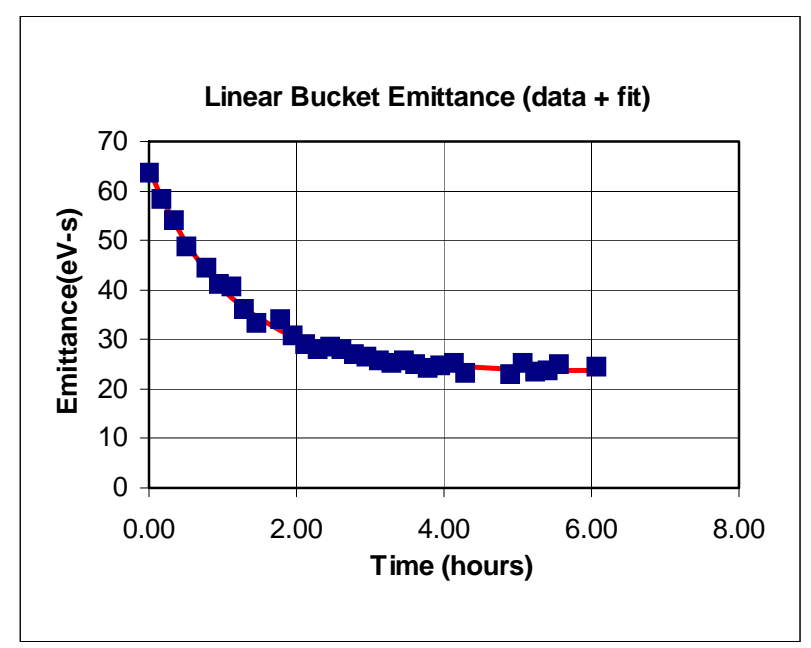

\section{DISCUSSION}

RR longitudinal stochastic cooling is obtained by notch filter cooling of the energy spread.[4] A general expression for energy cooling is:

$$
\frac{\mathrm{d} \sigma_{\mathrm{E}}^{2}}{\mathrm{dn}}=-2 \frac{\partial \frac{\mathrm{dE}}{\mathrm{dn}}}{\partial \mathrm{E}} \sigma_{\mathrm{E}}^{2}+\frac{\mathrm{d}\left\langle\Delta \mathrm{E}_{\mathrm{rms}}^{2}\right\rangle}{\mathrm{dn}}=-2 \lambda \sigma_{\mathrm{E}}^{2}+\mathrm{D}
$$

where the first term is the cooling term and the second is a heating term caused by various random processes that heat the beam. $\lambda$ is a damping coefficient, and D is a diffusion coefficient. With barrier bucket bunching the bunch length remains constant, the longitudinal emittance (the phase space area in energy spread and bunch-length $\delta \mathrm{E}-\delta \tau)$ is simply proportional to the energy spread. With linear bunching $\mathrm{rf}$, the energy spread and bunch length coordinates are continually mixed by harmonic motion:

$$
\frac{\mathrm{d}(\delta \mathrm{E})}{\mathrm{dn}}=\mathrm{eV}_{\mathrm{rf}}^{\prime} \delta \tau, \frac{\mathrm{d}(\delta \tau)}{\mathrm{dn}}=\frac{\alpha_{\mathrm{p}} \tau_{\mathrm{R}}}{\beta^{2} \mathrm{E}_{0}} \delta \mathrm{E},
$$

where $\alpha_{\mathrm{p}}=1 / \gamma^{2}-1 / \gamma_{\mathrm{t}}^{2}=0.0085, \tau_{\mathrm{R}}=11.35 \mu \mathrm{s}$ and $\mathrm{E}_{0}=$ $8.94 \mathrm{GeV}$ in the $\mathrm{RR}$, and the rate of change in $\sigma_{\mathrm{E}}$ is naturally half the coasting beam rate, with $\sigma_{\tau}$ changing at an equal rate. With matched harmonic motion, $\sigma_{\tau}=\beta_{\tau \mathrm{E}} \sigma_{\mathrm{E}}$, with $\beta_{\tau \mathrm{E}}=\left(\alpha_{\mathrm{p}} \tau_{\mathrm{R}} / \mathrm{eV}_{\mathrm{rf}} \beta^{2} \mathrm{E}_{0}\right)^{1 / 2}$.

The emittance cooling with linear rf becomes:

$\frac{\mathrm{d} \varepsilon_{\mathrm{L}}}{\mathrm{dn}}=-\frac{\partial \frac{\mathrm{dE}}{\mathrm{dn}}}{\partial \mathrm{E}} \varepsilon_{\mathrm{L}}+\frac{\beta_{\tau \mathrm{E}}}{2} \frac{\mathrm{d}\left\langle\Delta \mathrm{E}_{\mathrm{rms}}^{2}\right\rangle}{\mathrm{dn}}=-\lambda \varepsilon_{\mathrm{L}}+\frac{\beta_{\tau \mathrm{E}}}{2} \mathrm{D}$.

Because the bunch length is continuously reduced as the energy spread is reduced, the final emittance that can be obtained is smaller than that obtained with barrier-bucket cooling. This discussion assumes that the parameters $\lambda$ and $\mathrm{D}$ are constant and independent of the beam properties. This is in general not true for stochastic cooling, but is approximately true in this case.

\section{Particle mixing}

Stochastic cooling requires mixing of particles, so that independent particle samples are measured and corrected. However, a linear rf system has no synchrotron frequency spread. Averaging over synchrotron periods, particle trajectories have no frequency spread, and particle mixing is greatly suppressed. It was therefore somewhat surprising that the cooling rate was relatively unchanged in transforming from barrier bucket (with large synchrotron frequency spread) to a linear rf bucket.

To develop particle mixing, particle samples must be changed within the shielding time. (The shielding time is greater than the synchrotron period.) In the RR the cooling time, and the synchrotron period, are very long, and relatively small perturbations can provide mixing. (In the Tevatron bunched beam cooling the sample size and the bunch lengths were similar; in the RR the bunch length/sample length is $>10^{4}$.) In ref. [5] it is suggested that diffusive processes, such as intrabeam scattering (IBS) and multiple scattering, can randomize particle energies and positions sufficiently to enable mixing. The mixing time would be the time required for the diffusion to move a particle by the sample size; i. e. $\delta z \sim c /(2 \pi \mathrm{W})$ $\cong 0.03 \mathrm{~m}$, where $\mathrm{W}$ is the cooling band width $(1.5 \mathrm{GHz})$, and good mixing occurs if particle trajectories diffuse by the sample size within a synchrotron period. At RR parameters, the IBS scattering, with the synchrotron oscillation $\mathrm{rf}$, is $\sim 1000 \times$ the minimum needed to randomize particles by $\delta z$ within a synchrotron period. Multiple scattering is $\sim 20-50 \times$ the minimum.

Second-order frequency spread can also contribute to the mixing. $\left(\delta \mathrm{f} / \mathrm{f}_{0} \propto\left(\delta \mathrm{p}_{\mathrm{x}, \mathrm{y}, \mathrm{z}} / \mathrm{p}\right)^{2}\right)$

\section{Longitudinal heating}

The measured diffusion in the longitudinal cooling is somewhat larger than that expected from IBS and beamgas scattering, and also larger than expected from thermal noise and Shottky noise. The heating is reduced to $\sim$ IBS levels when the longitudinal cooling system is off, and must therefore be due to that cooling system. The present hypothesis is that it is primarily due to phase errors and nonlinear amplification in the notch-filter cooling system. The current notch filter is realized using a feedback 
stabilized fiber optic link. The characteristic frequency of the notch filter is set by using a network analyzer to tune the phase difference between short and long signal paths in the notch filter. The internal oscillator of the network analyzer is not phase-locked to the beam and the resulting systematic phase error can be equivalent to $\sim 10 \mathrm{ps}$ deviation from the revolution period. The difference between the notch filter characteristic frequency and the revolution frequency is equivalent to $\sim \delta p \cong 1 \mathrm{MeV} / \mathrm{c}$. The intrinsic dispersion of the notch filter will also introduce a spread $\delta \mathrm{p}>\sim 1 \mathrm{MeV} / \mathrm{c}$.

Intermodulational distortion from the TWT kicker amplifiers will decrease the effective notch depth. This effect is exacerbated by the systematic phase error. Since the beam is confined by the rf, the error signal developed by a notch filter with a systematic phase offset relative to the rf frequency will always be larger than minimal. These sources of diffusion in the stochastic cooling system result in an effective notch depth $10 \mathrm{~dB}$ less than that indicated by a network analysis of the notch filter. (A $\sim 10 \mathrm{~dB}$ enhancement of $\delta \mathrm{p} \cong 1 \mathrm{MeV} / \mathrm{c}$ noise could obtain the $\sim 3 \mathrm{MeV} / \mathrm{c} \delta \mathrm{p}$ noise seen in the RR). Future studies will attempt to improve the tuning of the cooling system to reduce the heating effect.

\section{OPERATIONAL IMPLEMENTATION}

Linear-rf bunched beam cooling is used in RR operation in order to minimize the longitudinal emittance of p-beam before injection into the Main Injector for the Tevatron Collider. The usual operational procedure is to capture p-beam within a barrier bucket, at a fixed bunch length,cool it transversely and longitudinally and then to introduce $\mathrm{a} \pm 2 \mathrm{kV}$ linear $\mathrm{rf}$, continuing with cooling. The rf pulse length is reduced (increasing the rf gradient) until a minimized longitudinal emittance is obtained. The resulting beam is then formed into trains of bunches for injection into the Main Injector for Collider use. The linear $\mathrm{rf}$ cooling enabled reducing the longitudinal emittance to the goal of $0.4 \times \mathrm{N}_{\mathrm{p}} / 10^{10} \mathrm{eV}$-s $\left(40 \mathrm{eV}-\mathrm{s}\right.$ at $\mathrm{N}_{\mathrm{p}}$ $=100 \times 10^{10}$ ). The cooled bunches extracted in this mode have contributed to the highest luminosities recorded in the Tevatron $\left(\mathrm{L}>10^{32} \mathrm{~cm}^{-2} \mathrm{~s}^{-1}\right)$. This has been the first successful operational application of bunched-beam stochastic cooling in high energy physics.

\section{FUTURE STUDIES AND PLANS}

At the present time, the longitudinal cooling system is being tuned to reduce heating and improve cooling, with bunched-beam cooling used to minimize the final longitudinal emittance. The operational practice has been to keep the energy spread approximately constant (at $\sigma_{\mathrm{E}} \cong$ $4 \mathrm{MeV}$ ), while decreasing the bunch length.

An electron cooling system is presently being commissioned for operational use in the Recycler.[7] That cooling system should be more effective in cooling longitudinally than the stochastic cooling system. When the electron cooling becomes fully effective, stochastic cooling will no longer be used to cool the beam longitudinally. The core transverse cooling will also be obtained through electron cooling, and stochastic cooling will only be used to reduce the large amplitude motion. The present procedure of gradually reducing the bunch length by increasing a linear-rf bunching gradient while cooling in energy will be adapted for use with electron cooling.

\section{REFERENCES}

[1] G. Jackson, "A Test of Bunched Beam Stochastic Cooling in the Fermilab Tevatron Collider", Proc. 1991 PAC, Washington, D. C., p. 1758 (1991).

[2] G. Jackson, "Bunched Beam Stochastic Cooling in the Fermilab Tevatron Collider", in Proc. Workshop on Beam Cooling, J. Bosser, ed. CERN 94-03, p.127 (1994).

[3] H. Herr and D. Mohl, "Bunched Beam Stochastic Cooling”, PS/DL/Note 79-3, EP/Note 79-34 (!979), in Proc. Workshop on Cooling of High Energy Beams (Madison, 1978).

[4] D. Broemmelsiek, M. Hu, and S. Nagaitsev, "Stochastic Cooling in Barrier Buckets in the Fermilab Recycler", Proc. EPAC2004, p.794 (2004).

[5] D. Broemmelsiek, A.Burov, D. Neuffer, S. Nagaitsev, "Diffusive Decoherence In Bunched-Beam Stochastic Cooling", submitted to PRSTAB (2004).

[6] V. Visnjic, "Intermodulation Distortion in the Stochastic Cooling System," PBAR-499, April, 1991.

[7] S. Nagaitsev, "Commissioning of Fermilab's Electron Cooling System for $8 \mathrm{GeV}$ Antiprotons", this proceedings (PAC2005).

Table 1: Parameters of the Fermilab recycler ring, with values matched to the cooling experiment.

\begin{tabular}{|l|c|c|c|}
\hline Parameter & Symbol & Value & Units \\
\hline \hline \multicolumn{3}{|c|}{ Fermilab Recycler Ring } \\
\hline Circumference & $C$ & 3320 & $\mathrm{~m}$ \\
\hline Momentum & $p$ & 8.9 & $\mathrm{GeV} / \mathrm{c}$ \\
\hline Slippage factor & $\eta$ & -0.0086 & \\
\hline Emittance $(\mathrm{n}, 95 \%)$ & $\varepsilon_{n}$ & $\sim 3$ & $\mu \mathrm{m}$ \\
\hline Average $\beta$-function & $\beta_{\text {ave }}$ & 30 & $\mathrm{~m}$ \\
\hline \multicolumn{3}{|c|}{ Momentum Cooling System } \\
\hline Number of pbars & $N$ & 30 & $10^{10}$ \\
\hline Bandwidth & $\Delta f$ & 1.5 & $\mathrm{GHz}$ \\
\hline Cooling time & $\tau$ & $\sim 1-2$ & hour \\
\hline Momentum spread & $\sigma_{p} / p$ & $\sim 3 \cdot 10^{-4}$ & $\mathrm{rms}$ \\
\hline
\end{tabular}

